\title{
Well-Posedness of Fixed Point Problem for a Multifunction Satisfying an Implicit Relation
}

\author{
Mohamed Akkouchi And Valeriu Popa
}

\begin{abstract}
The notion of well-posedness of a fixed point problem for a single valued mapping has generated much interest to a several mathematicians, for examples, F.S. De Blassi and J. Myjak (1989), S. Reich and A. J. Zaslavski (2001), B.K. Lahiri and P. Das (2005) and V. Popa (2006 and 2008). In this paper we extend the notion of well-posedness known for single valued mappings to the case of multifunctions. We establish the well-posedness of fixed point problem for a multifunction satisfying an implicit relation in orbitally complete metric spaces.
\end{abstract}

\section{INTRODUCTION}

Throughout this paper, $\mathbb{N}$ will be the set of non negative integers. Let $(X, d)$ be a metric space and $B(X)$ the set of all nonempty bounded sets of $X$. As in [6], [7] and [8], we define the functions $\delta(A, B)$ and $D(A, B)$ by

$$
\begin{aligned}
\delta(A, B) & :=\sup \{d(a, b): a \in A, b \in B\}, \\
D(A, B) & :=\inf \{d(a, b): a \in A, b \in B\} .
\end{aligned}
$$

If $A$ consists of single point " $a$ ", we write $\delta(A, B)=\delta(a, B)$. If $B$ consists of single point " $b$ ", we write $\delta(A, B)=\delta(A, b)$.

It follows immediately from the definition of $\delta(A, B)$ that

$$
\delta(A, B)=\delta(B, A), \quad \forall A, B \in B(X),
$$

and

$$
\delta(A, B) \leq \delta(A, C)+\delta(C, B), \quad \forall A, B, C \in B(X) .
$$

Definition 1.1. A sequence $\left\{A_{n}\right\}$ of nonempty subsets of $X$ is said to converge to a subset $A$ of $X$ if:

(i) Each point $a \in A$ is the limit of a convergent sequence $\left\{a_{n}\right\}$, where $a_{n} \in A_{n}$, for all $n \in \mathbb{N}$.

1991 Mathematics Subject Classification. Primary: 54H25, 47H10.

Key words and phrases. Well-posedness of fixed point problem for a multifunction, strict fixed points, implicit relations, orbitally complete metric spaces. 
(ii) For arbitrary $\epsilon>0$ there exists an integer $m>0$ such that $A_{n} \subset$ $A(\epsilon)$, where

$$
A(\epsilon):=\{x \in X: \exists a \in A: d(x, a)<\epsilon\} .
$$

The set $A$ is said to be the limit of the sequence $\left\{A_{n}\right\}$.

Lemma 1.1 (Fisher [6]). If $\left\{A_{n}\right\}$ and $\left\{B_{n}\right\}$ are two sequences in $B(X)$ converging to the sets $A$ and $B$ respectively in $B(X)$, then the sequence $\left\{\delta\left(A_{n}, B_{n}\right)\right\}$ converges to $\delta(A, B)$.

Lemma 1.2 (Fisher and Sessa [8]). Let $\left\{A_{n}\right\}$ be a sequence in $B(X)$ and $y \in X$ such that $\lim _{n \rightarrow \infty} \delta\left(A_{n}, y\right)=0$. Then the sequence $\left\{A_{n}\right\}$ converges to $\{y\}$ in $B(X)$.

Definition 1.2. Let $F: X \rightarrow B(X)$ be a multifunction.

a) A point $x \in X$ is a fixed point of $F$ if $x \in F x$.

b) A point $x \in X$ is a strict fixed point of $F$ if $\{x\}=F x$.

The importance of orbits of points under self-mappings in metric spaces is well recognized. In many early papers dealing with fixed point theory, the orbits were used to investigate fixed points. (See for example [5], [3] and others).

We recall the following definition (see for instance [2], [3] and others).

Definition 1.3. Let $f:(X, d) \rightarrow(X, d)$. If for any $x \in X$, every Cauchy sequence of the orbit $O(f, x):=\left\{x, f x, f^{2} x, \ldots\right\}$ is convergent in $X$, then the metric space is said to be $f$-orbitally complete.

Remark 1.1. Every complete metric space is $f$-orbitally complete for any f. An orbitally complete space may not be complete metric space (see [15]).

Let $F: X \rightarrow B(X)$ and $x_{0} \in X$. An orbit of $F$ at point $x_{0}$, is a sequence $\left\{x_{n}\right\}$ given by

$$
O\left(F, x_{0}\right):=\left\{x_{n}: x_{n+1} \in F\left(x_{n}\right), n=0,1,2, \ldots\right\} .
$$

Definition 1.4. Let $(X, d)$ be a metric space. Let $F: X \rightarrow B(X)$ be a multifunction. $(X, d)$ is called to be $F$-orbitally complete, if for all $x \in X$, every Cauchy subsequence of the orbit $O(F, x)$ converges to a point in $X$.

The notion of well-posedness of a fixed point problem has evoked much interest to several mathematicians (see for example [14], [4], [9], [12], [13] and [1]).

Definition 1.5. Let $(X, d)$ be a metric space and $f:(X, d) \rightarrow(X, d)$ be a mapping. The fixed point problem of $f$ is said to be well posed if:

(i) $f$ has a unique fixed point $z$ in $X$,

(ii) for any sequence $\left\{x_{n}\right\}$ of points in $X$ such that $\lim _{n \rightarrow \infty} d\left(T x_{n}, x_{n}\right)=$ 0 , we have $\lim _{n \rightarrow \infty} d\left(x_{n}, z\right)=0$. 
We extend Definition 1.5 for multifunctions.

Definition 1.6. Let $(X, d)$ be a metric space and $F: X \rightarrow B(X)$ be a multifunction. The fixed point problem of $F$ is said to be well-posed if:

(i) $F$ has a unique strict fixed point $z$ in $X$,

(ii) for any sequence $\left\{x_{n}\right\}$ of points in $X$ such that $\lim _{n \rightarrow \infty} \delta\left(F x_{n}, x_{n}\right)=$ 0 , we have $\lim _{n \rightarrow \infty} d\left(x_{n}, z\right)=0$.

The study of fixed point for mappings satisfying an implicit relation is initiated and studied in [10] and [11].

In this paper we prove a general fixed point theorem for multifunctions satisfying an implicit relation in orbitally complete metric spaces and that fixed point problem is well-posed generalizing some results from [1] and [9].

\section{IMPLICIT RELATIONS}

Let $\phi\left(t_{1}, \ldots, t_{6}\right): \mathbb{R}^{6} \rightarrow \mathbb{R}$ be a continuous function. We define the following properties:

$\left(\phi_{1}\right): \phi$ is non-increasing in the variables $t_{2}, t_{5}$ and $t_{6}$ and non-decreasing in the variable $t_{1}$.

$\left(\phi_{2}\right)$ : There exists a real number $h \in(0,1)$ such that for every $u \geq 0$, $v \geq 0$ with $\phi(u, v, v, u, u+v, 0) \leq 0$, we have $u \leq h v$.

$\left(\phi_{3}\right): \phi(t, t, 0,0, t, t)>0$, for every $t>0$.

$\left(\phi_{p}\right)$ : There exists $p \in(0,1)$ such that for every $u \geq 0, v \geq 0, w \geq 0$ with $\phi(u, v, 0, w, u, v) \leq 0$, we have $u \leq p \max \{v, w\}$.

Example 2.1. $\phi\left(t_{1}, \ldots, t_{6}\right)=t_{1}-c \max \left\{t_{2}, t_{3}, t_{4}, \frac{1}{2}\left(t_{5}+t_{6}\right)\right\}$, where $c \in$ $(0,1)$.

$\left(\phi_{1}\right)$ : Obviously.

$\left(\phi_{2}\right)$ : For all $u, v \geq 0$, we have

$$
\phi(u, v, v, u, u+v, 0)=u-c \max \left\{u, v, \frac{1}{2}(u+v)\right\}=u-c \max \{u, v\} .
$$

Suppose that $\phi(u, v, v, u, u+v, 0) \leq 0$ and that $u>v$. Then, from (2.1), we get $u(1-c) \leq 0$, a contradiction. Therefore $u \leq v$, which yields (by $(2.1)$ ) that $u \leq c v$. Thus $\left(\phi_{2}\right)$ is true with $h:=c \in(0,1)$. $\left(\phi_{3}\right): \phi(t, t, 0,0, t, t)=t(1-c)>0$ for all $t>0$.

$\left(\phi_{p}\right)$ : For all $u, v, w \geq 0$, we have

$$
\phi(u, v, 0, w, u, v)=u-c \max \left\{v, w, \frac{1}{2}(u+v)\right\} .
$$

Suppose that $\phi(u, v, 0, w, u, v) \leq 0$, with $u>0$ and $u \geq \max \{v, w\}$. Then we have $u(1-c) \leq 0$, a contradiction. Hence, $0<u \leq$ $\max \{v, w\}$, which implies that $\frac{1}{2}(u+v) \leq \max \{v, w\}$. Thus, we get $u \leq c \max \{v, w\}$. If $u=0$, then $u \leq c \max \{v, w\}$. This shows that $\left(\phi_{p}\right)$ is true with $p:=c \in(0,1)$. 
Example 2.2. $\phi\left(t_{1}, \ldots, t_{6}\right)=t_{1}-a_{1} t_{2}-a_{2} t_{3}-a_{3} t_{4}-a_{4} t_{5}-a_{5} t_{6}$, where $a_{i} \geq 0$ for $i=1,2, \ldots, 5, a_{1}+a_{3}+a_{5}>0,0<a_{1}+a_{3}+a_{4}+a_{5}<1$ and $0<a_{1}+a_{2}+a_{3}+2 a_{4}<1$.

$\left(\phi_{1}\right)$ : Obviously.

$\left(\phi_{2}\right)$ : For all $u, v \geq 0$, we have

$$
\phi(u, v, v, u, u+v, 0)=u\left(1-a_{3}-a_{4}\right)-v\left(a_{1}+a_{2}+a_{4}\right) .
$$

If $\phi(u, v, v, u, u+v, 0) \leq 0$, then $u \leq h v$, where $h:=\frac{a_{1}+a_{2}+a_{4}}{1-a_{3}-a_{4}}$. By assumptions, we have $h \in(0,1)$.

$\left(\phi_{3}\right): \phi(t, t, 0,0, t, t)=t\left(1-a_{1}-a_{4}-a_{5}\right)>0$ for all $t>0$.

$\left(\phi_{p}\right)$ : For all $u, v, w \geq 0$, we have

$$
\phi(u, v, 0, w, u, v)=u\left(1-a_{4}\right)-v\left(a_{1}+a_{5}\right)-a_{3} w .
$$

Suppose that $\phi(u, v, 0, w, u, v) \leq 0$, then

$$
u\left(1-a_{4}\right) \leq v\left(a_{1}+a_{5}\right)+a_{3} w \leq\left(a_{1}+a_{3}+a_{5}\right) \max \{v, w\} .
$$

Thus $u \leq p \max \{v, w\}$, where $p:=\frac{a_{1}+a_{3}+a_{5}}{1-a_{4}}$. By assumptions, we have $p \in(0,1)$.

Example 2.3. $\phi\left(t_{1}, \ldots, t_{6}\right)=t_{1}^{2}-a t_{2} t_{3}-b t_{3} t_{4}-c t_{5} t_{6}$, where $a>0, b, c \geq 0$, $a+b<1$ and $a+c<1$.

$\left(\phi_{1}\right)$ : Obviously.

$\left(\phi_{2}\right)$ : For all $u, v \geq 0$, we have

$$
\phi(u, v, v, u, u+v, 0)=u^{2}-a v^{2}-b u v .
$$

Let $v>0$ and $f(t)=t^{2}-b t-a$, where $t=\frac{u}{v}$. We observe that $f(0)=-a<0$ and $f(1)=1-(a+b)>0$. Then there exists $h \in(0,1)$ suc that $f(h)=0$. Since the other root of the equation $f(t)=0$ is strictly negative, then the inequality $f(t) \leq 0(t \geq 0)$ implies that $t \leq h$. Thus, if $\phi(u, v, v, u, u+v, 0) \leq 0$ with $v>0$, then we have $u \leq h v$. If $v=0$, then from (2.3) we get $u=0$. Therefore $u \leq h v$.

$\left(\phi_{3}\right): \phi(t, t, 0,0, t, t)=t^{2}(1-c)>0$ for all $t>0$.

$\left(\phi_{p}\right)$ : For all $u, v, w \geq 0$, we have

$$
\phi(u, v, 0, w, u, v)=u^{2}-c u v .
$$

Suppose that $\phi(u, v, 0, w, u, v) \leq 0$ and $u>0$. Then we obtain $u \leq c v \leq \max \{v, w\}$. Thus $u \leq p \max \{v, w\}$, where $p:=c \in(0,1)$. If $u=0$, then $u \leq p \max \{v, w\}$. This shows that $\left(\phi_{p}\right)$ is satisfied.

Example 2.4. $\phi\left(t_{1}, \ldots, t_{6}\right)=t_{1}-a t_{2}-b t_{3}-c t_{4}-d \min \left\{t_{5}, t_{6}\right\}$, where $a, b, c \geq 0,0<a+b \leq a+b+c<1$ and $0<a+c+d<1$.

$\left(\phi_{1}\right)$ : Obviously. 
$\left(\phi_{2}\right)$ : For all $u, v \geq 0$, we have

$$
\phi(u, v, v, u, u+v, 0)=u(1-c)-v(a+b) .
$$

Suppose that $\phi(u, v, v, u, u+v, 0) \leq 0$. Then $u \leq h v$, where $h:=$ $\frac{a+b}{1-c} \in(0,1)$. Hence $\left(\phi_{2}\right)$ is satisfied.

$\left(\phi_{u}\right): \phi(t, t, 0,0, t, t)=t(1-a-d)>0$ for all $t>0$.

$\left(\phi_{p}\right)$ : For all $u, v, w \geq 0$, we have

$$
\phi(u, v, 0, w, u, v)=u-a v-c w-d \min \{u, v\} .
$$

If $\phi(u, v, 0, w, u, v) \leq 0$ and $u>\max \{u, v\}$, then we obtain $u(1-$ $a-c-d) \leq 0$, a contradiction. Hence $u \leq \max \{v, w\}$, and then $u \leq p \max \{v, w\}$, where $p:=a+c+d \in(0,1)$. This proves that $\left(\phi_{p}\right)$ is satisfied.

Example 2.5. $\phi\left(t_{1}, \ldots, t_{6}\right)=t_{1}-c \max \left\{t_{2}, t_{3}, \sqrt{t_{4} t_{6}}, \sqrt{t_{5} t_{6}}\right\}$, where $0<$ $c<1$.

$\left(\phi_{1}\right)$ : Obviously.

$\left(\phi_{2}\right)$ : For all $u, v \geq 0$, we have

$$
\phi(u, v, v, u, u+v, 0)=u-c v .
$$

If $\phi(u, v, v, u, u+v, 0) \leq 0$, then $u \leq h v$, where $h:=c \in(0,1)$. $\left(\phi_{3}\right): \phi(t, t, 0,0, t, t)=t(1-c)>0$ for all $t>0$. Because $c \in(0,1)$. $\left(\phi_{p}\right)$ : For all $u, v, w \geq 0$, we have

$$
\phi(u, v, 0, w, u, v)=u-c \max \{v, \sqrt{w v}, \sqrt{u v}\} .
$$

If $\phi(u, v, 0, w, u, v) \leq 0$ and $u>\max \{v, w\}$, then we obtain $u(1-c) \leq$ 0 , a contradiction. Hence $u \leq \max \{v, w\}$ and therefore we have $u \leq p \max \{v, w\}$, where $p:=c \in(0,1)$. This proves that $\left(\phi_{p}\right)$ is satisfied.

Remark 2.1. There exists $\phi: \mathbb{R}^{6} \rightarrow \mathbb{R}$ increasing in variables $t_{3}, t_{4}$ which satisfies properties $\left(\phi_{1}\right),\left(\phi_{2}\right),\left(\phi_{3}\right)$ and $\left(\phi_{p}\right)$.

Example 2.6. $\phi\left(t_{1}, \ldots, t_{6}\right)=t_{1}^{2}-a t_{2}^{2}-b \frac{t_{5} t_{6}}{1+t_{3}+t_{4}}$, where $a>0, b \geq 0$ and $0<a+b<1$.

$\left(\phi_{1}\right)$ : Obviously.

$\left(\phi_{2}\right)$ : For all $u, v \geq 0$, we have

$$
\phi(u, v, v, u, u+v, 0)=u^{2}-a v^{2} .
$$

If $\phi(u, v, v, u, u+v, 0) \leq 0$, then $u \leq h v$, where $h:=a \in(0,1)$. Hence $\left(\phi_{2}\right)$ is satisfied.

$\left(\phi_{3}\right): \phi(t, t, 0,0, t, t)=t^{2}(1-a-b)>0$ for all $t>0$.

$\left(\phi_{p}\right)$ : For all $u, v, w \geq 0$, we have

$$
\phi(u, v, 0, w, u, v)=u^{2}-a v^{2}-b \frac{u v}{1+w} .
$$


If $\phi(u, v, 0, w, u, v) \leq 0$, then $u^{2}-a v^{2}-b u v \leq 0$. As in the proof of $\left(\phi_{2}\right)$ in Example 2.3, we obtain $u \leq p \max \{v, w\}$, for some $p \in(0,1)$. This proves that $\left(\phi_{p}\right)$ is satisfied.

\section{MAIN RESUlts}

Theorem 3.1. Let $(X, d)$ be a metric space and $F: X \rightarrow B(X)$ a mulifunction such that

$$
\phi(\delta(F x, F y), d(x, y), \delta(x, F x), \delta(y, F y), D(x, F y), D(y, F x)) \leq 0,
$$

for all $x, y \in X$, where $\phi$ satisfies property ( $\left.\phi_{3}\right)$, then $F$ has at most one strict fixed point in $X$.

Proof. Suppose that $z$ and $y$ are strict fixed points of $F$ with $z \neq y$. Then $\{z\}=F z$ and $\{y\}=F y$. By (3.1) we obtain

$$
\begin{aligned}
\phi(\delta(F z, F y), d(z, y), \delta(z, F z), \delta(y, F y), D(z, F y), D(y, F z)) & = \\
=\phi(d(z, y), d(z, y), 0,0, d(z, y), d(z, y)) & \leq 0
\end{aligned}
$$

a contradiction of $\left(\phi_{3}\right)$.

Theorem 3.2. Let $(X, d)$ be a metric space and $F: X \rightarrow B(X)$ a mulifunction such that

$$
\phi(\delta(F x, F y), d(x, y), \delta(x, F x), \delta(y, F y), D(x, F y), D(y, F x)) \leq 0,
$$

for all $x, y \in X$, where $\phi$ satisfies properties $\left(\phi_{1}\right),\left(\phi_{2}\right)$ and $\left(\phi_{3}\right)$. Then $F$ has an unique fixed point in $X$ which is strict fixed point for $F$.

Proof. Let $x_{0}$ be any arbitrary point in $X$ and consider the orbit of $F$ at $x_{0}$ given by the sequence $\left\{x_{n}\right\}$ such that $x_{n+1} \in F x_{n}$ for all integers $n=$ $0,1,2, \ldots$ Then by $(3.1)$, we have

$$
\begin{aligned}
& \phi\left(\delta\left(F x_{n}, F x_{n+1}\right), d\left(x_{n}, x_{n+1}\right), \delta\left(x_{n}, F x_{n}\right),\right. \\
& \left.\quad \delta\left(x_{n+1}, F x_{n+1}\right), D\left(x_{n}, F x_{n+1}\right), D\left(x_{n+1}, F x_{n}\right)\right) \leq 0
\end{aligned}
$$

Since $D\left(x_{n+1}, F x_{n}\right)=0, \delta\left(F x_{n}, F x_{n+1}\right) \geq \delta\left(x_{n+1}, F x_{n+1}\right)$ and $\phi$ in nondecreasing in the variable $t_{1}$ then we have

$\phi\left(\delta\left(x_{n+1}, F x_{n+1}\right), d\left(x_{n}, x_{n+1}\right), \delta\left(x_{n}, F x_{n}\right), \delta\left(x_{n+1}, F x_{n+1}\right), D\left(x_{n}, F x_{n+1}\right), 0\right) \leq 0$.

Since $d\left(x_{n}, x_{n+1}\right) \leq \delta\left(x_{n}, F x_{n}\right), D\left(x_{n}, F x_{n+1}\right) \leq d\left(x_{n}, x_{n+1}\right)+\delta\left(x_{n+1}, F x_{n+1}\right)$ and $\phi$ is non-increasing in the variables $t_{2}$ and $t_{5}$ then we get

$$
\begin{aligned}
\phi\left(\delta\left(x_{n+1}, F x_{n+1}\right), \delta\left(x_{n}, F x_{n}\right), \delta\left(x_{n}, F x_{n}\right),\right. \\
\left.\quad \delta\left(x_{n+1}, F x_{n+1}\right), \delta\left(x_{n}, F x_{n}\right)+\delta\left(x_{n+1}, F x_{n+1}\right), 0\right) \leq 0 .
\end{aligned}
$$

By property $\left(\phi_{2}\right)$, we have $\delta\left(x_{n+1}, F x_{n+1}\right) \leq h \delta\left(x_{n}, F x_{n}\right)$ and so

$$
\delta\left(x_{n}, F x_{n}\right) \leq h^{n} \delta\left(x_{0}, F x_{0}\right), \quad \forall n \geq 0 .
$$


(3.2) shows that the sequence $\left\{\delta\left(x_{n}, F x_{n}\right)\right\}$ is a strongly Cauchy sequence (that is $\sum_{n \geq 0} \delta\left(x_{n}, F x_{n}\right)$ converges). Since $d\left(x_{n}, x_{n+1}\right) \leq \delta\left(x_{n}, F x_{n}\right)$, then the sequence $\left\{d\left(x_{n}, x_{n+1}\right)\right\}$ is also a strongly Cauchy sequence. It follows that $\left\{x_{n}\right\}$ is a Cauchy sequence in the orbit $O\left(F, x_{0}\right)$. Since $(X, d)$ is $F$ orbitally complete, the sequence $\left\{x_{n}\right\}$ is convergent to a point $z \in X$. We prove that $\{z\}=F z$. For each positive integer $n$, we have

$$
\delta\left(F x_{n}, z\right) \leq \delta\left(F x_{n}, x_{n}\right)+d\left(x_{n}, z\right) .
$$

By (3.2) we obtain that $\lim _{n \rightarrow \infty} \delta\left(F x_{n}, z\right)=0$. Then by Lemma 1.2, the sequence $\left\{F x_{n}\right\}$ converges to the set $\{z\}$ in $B(X)$. By the inequality (3.1) for $x:=x_{n}$ and $y:=z$, we obtain

$$
\phi\left(\delta\left(F x_{n}, F z\right), d\left(x_{n}, z\right), \delta\left(x_{n}, F x_{n}\right), \delta(z, F z), D\left(x_{n}, F z\right), D\left(z, F x_{n}\right)\right) \leq 0,
$$

which (since $\phi$ is non-increasing in variables $\left.t_{5}, t_{6}\right)$ implies

$$
\phi\left(\delta\left(F x_{n}, F z\right), d\left(x_{n}, z\right), \delta\left(x_{n}, F x_{n}\right), \delta(z, F z), \delta\left(x_{n}, F z\right), \delta\left(z, F x_{n}\right)\right) \leq 0 .
$$

Letting $n$ tend to infinity we obtain

$$
\phi(\delta(z, F z), 0,0, \delta(z, F z), \delta(z, F z), 0)) \leq 0 .
$$

By property $\left(\phi_{2}\right)$, we obtain $\delta(z, F z)=0$, i.e., $\{z\}=F z$. Therefore $z$ is a strict fixed point for $F$. By Theorem 3.1, $z$ is the unique stricte fixed point for $F$. This completes the proof.

If $F$ is single-valued, Then the proof of Theorem 3.1 does not need the assumption $\left(\phi_{1}\right)$. So we recapture Theorem 3.1 of [1].

Corollary 3.1 (Theorem $3.1[1])$. Let $(X, d)$ be a metric space and let $T$ : $X \rightarrow X$ be a self-mapping. Suppose that $(X, d)$ is $T$-orbitally complete and that $T$ satisfies the inequality

$$
\phi(d(T x, T y), d(x, y), d(x, T x), d(y, T y), d(x, T y), d(y, T x)) \leq 0,
$$

for all $x, y \in X$, where $\phi$ satisfies properties $\left(\phi_{2}\right)$ and $\left(\phi_{3}\right)$. Then $T$ has a unique fixed point in $X$.

Theorem 3.3. Let $(X, d)$ be a metric space and $F: X \rightarrow B(X)$ a mulifunction such that

$$
\phi(\delta(F x, F y), d(x, y), \delta(x, F x), \delta(y, F y), D(x, F y), D(y, F x)) \leq 0,
$$

for all $x, y \in X$, where $\phi$ satisfies properties $\left(\phi_{1}\right),\left(\phi_{2}\right),\left(\phi_{3}\right)$ and $\left(\phi_{p}\right)$. Then the fixed point problem for $F$ is well posed.

Proof. By Theorem 3.2, $F$ has a unique strict fixed point $z$. Let $\left\{x_{n}\right\}$ be a sequence in $X$ such that

$$
\lim _{n \rightarrow \infty} \delta\left(x_{n}, F x_{n}\right)=0 .
$$

By inequality (3.1) we obtain

$$
\phi\left(\delta\left(F z, F x_{n}\right), d\left(z, x_{n}\right), \delta(z, F z), \delta\left(x_{n}, F x_{n}\right), D\left(z, F x_{n}\right), D\left(x_{n}, F z\right)\right) \leq 0 .
$$


Since $\{z\}=F z$, the previous inequality is equivalent to the following

$$
\phi\left(\delta\left(z, F x_{n}\right), d\left(z, x_{n}\right), 0, \delta\left(x_{n}, F x_{n}\right), D\left(z, F x_{n}\right), d\left(x_{n}, z\right)\right) \leq 0 .
$$

Since $\phi$ is non-increasing in the variable $t_{5}$, then we have

$$
\phi\left(\delta\left(z, F x_{n}\right), d\left(z, x_{n}\right), 0, \delta\left(x_{n}, F x_{n}\right), \delta\left(z, F x_{n}\right), d\left(x_{n}, z\right)\right) \leq 0 .
$$

By $\left(\phi_{p}\right)$, we have

$$
\delta\left(z, F x_{n}\right) \leq p \max \left\{d\left(z, x_{n}\right), \delta\left(x_{n}, F x_{n}\right)\right\} .
$$

On the other hand, we have

$$
d\left(z, x_{n}\right) \leq \delta\left(z, F x_{n}\right)+\delta\left(F x_{n}, x_{n}\right) \leq p\left[d\left(z, x_{n}\right), \delta\left(x_{n}, F x_{n}\right)\right]+\delta\left(F x_{n}, x_{n}\right),
$$

which implies that

$$
d\left(z, x_{n}\right) \leq \frac{1+p}{1-p} \delta\left(x_{n}, F x_{n}\right) \quad \rightarrow 0 \quad \text { as } \quad n \rightarrow \infty .
$$

Hence $\lim _{n \rightarrow \infty} d\left(z, x_{n}\right)=0$ and the fixed point problem of $F$ is well-posed.

As a consequence, we have the following result.

Corollary 3.2 (Theorem $3.2[1])$. Let $(X, d)$ be a metric space and let $T$ : $X \rightarrow X$ be a self-mapping. Suppose that $(X, d)$ is T-orbitally complete and that $T$ satisfies the inequality

$$
\phi(d(T x, T y), d(x, y), d(x, T x), d(y, T y), d(x, T y), d(y, T x)) \leq 0,
$$

for all $x, y \in X$, where $\phi$ satisfies properties $\left(\phi_{2}\right)$, ( $\left.\phi_{3}\right)$ and $\left(\phi_{p}\right)$. Then the fixed point problem of $T$ is well-posed.

Acknowledgement. The authors thank very much the anonymous referee for helpful comments.

\section{REFERENCES}

[1] M. Akkouchi and V. Popa, Well-posedness of fixed point problem for mappings satisfying an implicit relation, Demonstratio Math., 43(4) (2010), 923-929.

[2] Lj.B. Ćirić, On contraction type mappings, Math. Balkanica, 1 (1971), 52-57.

[3] Lj.B. Ćirić, On some maps with non-unique fixed points, Publ. Inst. Math. (Beograd), 17 (31) (1974), 52-58.

[4] F.S. De Blassi and J. Myjak, Sur la porosite des contractions sans point fixe, Comptes Rendus Academie Sciences Paris, (308), pp. 51-54, 1989.

[5] M. Edelstein, An extension of Banach's contraction principle, Proc. Amer. Math. Soc. 12 (1961), 7-10.

[6] B. Fisher, Common fixed points of mappings and set valued mappings, Rostock Math. Kollq, 18 (1981), 69-77.

[7] B. Fisher, Common fixed points on a metric space, Kyungpook Math. J., 25 (1985), 35-42. 
[8] B. Fisher and S. Sessa, Two common fixed point theorems for weakly commuting mappings, Periodica Math. Hungarica, 20(3) (1989), 207-218.

[9] B.K. Lahiri and P. Das, Well-posednes and porosity of certain classes of operators, Demonstratio Math., (38) (2005), 170-176.

[10] V. Popa, Fixed point theorems for implicit contractive mappings, Stud. Cerc. St. Ser. Mat. Univ. Bacău, 7 (1997), 129-133.

[11] V. Popa, Some fixed point theorems for compatible mappings satisfying an implicit relation, Demonstratio Math. 32 (1999), 157-163.

[12] V. Popa, Well-posedness of fixed point problem in orbitally complete metric spaces, Stud. Cerc. St. Ser. Mat. Univ. Bacău, 16 (2006), Suppl. 209-214.

[13] V. Popa, Well-Posedness of Fixed Point Problem in Compact Metric Spaces, Bul. Univ. Petrol-Gaze, Ploiesti, Sec. Mat. Inform. Fiz. 60, 1 (2008), 1-4.

[14] S. Reich and A.J. Zaslavski, Well-posednes of fixed point problems, Far East Journal Mathematical Sciences, Special volume 2001, Part III, pp. 393-401, 2001.

[15] D. Turkoglu, O. Ozer and B. Fisher, Fixed point theorems for T-orbitally complete spaces, Stud. Cerc. St. Ser. Mat., Univ. Bacău, 9 (1999), 211-218.

Mohamed Akmouchi

Université CAdi Ayyad

Faculté des Sciences-Semlalia

Département de Mathématiques

Av. Prince My Abdellah, BP. 2390

MARRAKECH

MorocCO

E-mail address: akkouchimo@yahoo.fr

\section{VAleriu Popa}

Universitatea Vasile Alecsandri

Str. Spiru Haret NR. 8

600114, BACĂU

ROMANIA

E-mail address: vpopa@ub.ro 